\title{
Mechanical behaviour modelling under dynamic conditions: Application to structural and high strength steels
}

\author{
Pierre Simon ${ }^{1,2, *}$, Yaël Demarty ${ }^{1}$, and Alexis Rusinek ${ }^{2}$ \\ ${ }^{1}$ French-German research institute of Saint-Louis, 5 rue du general Cassagnou, 68300 Saint-Louis, France \\ ${ }^{2}$ Laboratory of Microstructure Studies and Mechanics of Materials, UMR-CNRS 7239, Lorraine University, 7 rue Félix Savart, BP \\ 15082, 57073 Metz Cedex 03, France
}

This work is in the framework of a PhD partially funded by the Direction General de l'Armement.

\begin{abstract}
Current needs in the design and optimization of complex ballistic protection structures lead to the development of more and more accurate numerical modelling for high impact velocity. The aim of developing such a tool is to be able to predict the protection performance of structures using few experiments. Considering only numerical approach, most important issue to have a reliable simulation is to focus on material behaviour description in term of constitutive relation and failure model for high strain rates, large field of temperatures and complex stress states. In this context, the study deals with the behaviour of two steels including a high strength steel and a structural steel. For this application, the materials can undergo both quasi-static and dynamic loading. Thus the strain rate range studied is varying from $10^{-3}$ to more than $10^{3} \mathrm{~s}^{-1}$. Although the high strength steels do not exhibit high strain rate sensitivity, the temperature increases during dynamic loading is inducing thermal softening. Thus, temperature sensitivity is defined up to $500 \mathrm{~K}$ under quasi-static and dynamic conditions. Then, experiments are used to define the parameters of several constitutive relations like the Johnson-Cook model or the Rusinek Klepaczko model.
\end{abstract}

\section{Introduction}

The development of protective systems against ballistic impacts usually requires the use of several materials leading to complex structures. The design and optimization of such multi-layered structures is made possible through the use of numerical simulations. In fact, the number of parameters to consider (thickness, position of the materials, type of materials, etc.) is too significant to perform a parametric study using expensive and time consuming experimental campaigns.

Nevertheless, during experiment real mechanical material behaviour can be directly observed whereas in numerical model constitutive relations are used to describe it. Therefore, reliability of these relations is essential to obtain valid results. These material models usually represent the equivalent plastic stress of a material as a function of equivalent plastic strain, strain rate and temperature. Many relations have been developed through last decades, and are still under consideration. One can distinguish two main types of constitutive relations. The first ones are phenomenological, as they do not take into account any physical phenomenon and are only based on experimental observation. These relations have the main advantage of a low number of parameters. Nevertheless, their validity is most of time reduce at a small range of condition. A well-known and widely implemented example of phenomenological model used for metals is the Johnson-Cook constitutive model [1]. The second category is semi-physical constitutive relations. Although these models are more complicate to handle because of their higher number of parameters, they allow a better representation of material behaviour and an extended range of validity, as they take into account physical consideration.

This study focuses on the dynamic mechanical behaviour of two distinct steels. The first one is a structural steel, while the second-one is a high strength steel which could be used as an armour steel. In a first part of this paper, the sensitivities to strain rates and temperature are studied. For this purpose, quasi-static and Hopkinson bar experiments were conducted. Positive and negative (only in dynamic regime) temperatures have been applied. The second part deals with the material modelling. Different constitutive models are presented and the identification of their related parameters is detailed. The third part shows a comparative study of the different models with the experimental results.

\section{Experimental study}

Corresponding author: simon.pierre57@gmail.com 
In order to determine the hardening, the strain rate and temperature sensitivities, compression tests have been performed. The studied range of strain rate is varying from $10^{-3} \mathrm{~s}^{-1}$ to more than $10^{3} \mathrm{~s}^{-1}$. Regarding the temperature, experiments allowed to observe material behaviour up to $473 \mathrm{~K}$ in both quasi-static and dynamic cases. Experiments at low temperature (down to $173 \mathrm{~K}$ ) were also carried out in dynamic regime, using liquid nitrogen with addition of ethanol to adjust the temperature. Experiments at low strain rate from $10^{-3}$ to $10^{-1} \mathrm{~s}^{-1}$ have been performed using a quasi-static press, while higher strain rates have been reached using split Hopkinson pressure bar technique.

\subsection{Experiments at various strain rate}

The stress value for each strain rate and assuming a strain level is represented, Fig. 1 and Fig. 2, for each steels. A strain value below 0.1 was chosen to reduce the adiabatic heating effect on the stress value. In both cases, the stress increases non-linearly with the strain rate.

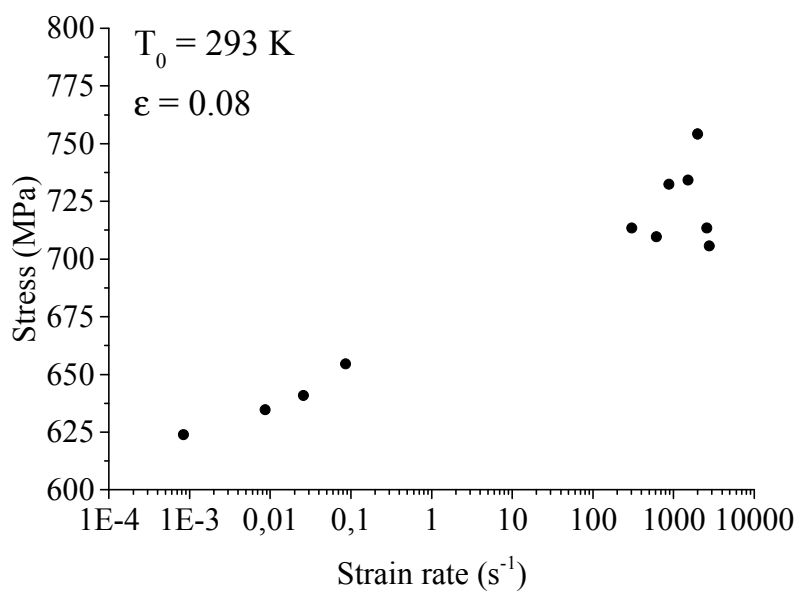

Fig. 1. Stress vs. strain rate (structural steel).

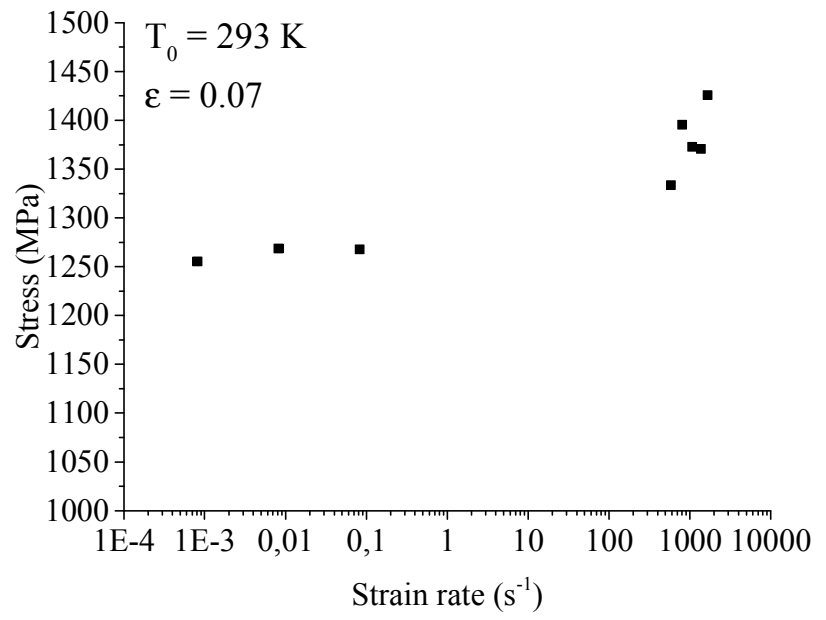

Fig. 2. Stress vs. strain rate (high strength steel).

\subsection{Experiments at various temperature}

Regarding experiments at high temperatures, each sample and the end of the bars have been heated for 30 minutes before the test to have a homogeneous temperature distribution in the specimen [2]. The stress values for each temperature and a strain level imposed are reported Fig. 3 and Fig. 4. The stress decreases as the temperature increase. As for the strain rate sensitivity, the structural steel presents higher temperature sensitivity in comparison with the high strength steel.

\section{Constitutive models}

In this part, models are compared with experiments reported previously. It allows observing if the models are able to describe the material behaviour.

\subsection{Johnson-Cook constitutive model}

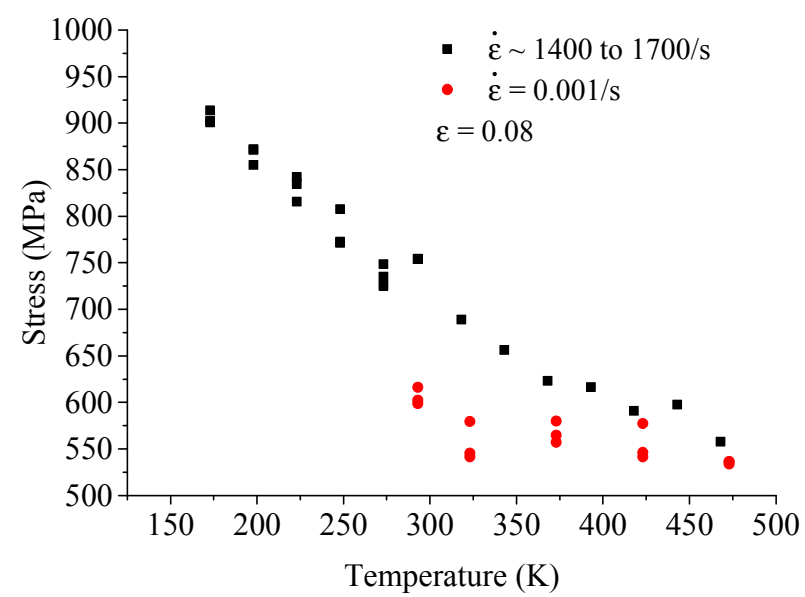

Fig. 3. Stress vs. Temperature (structural steel).

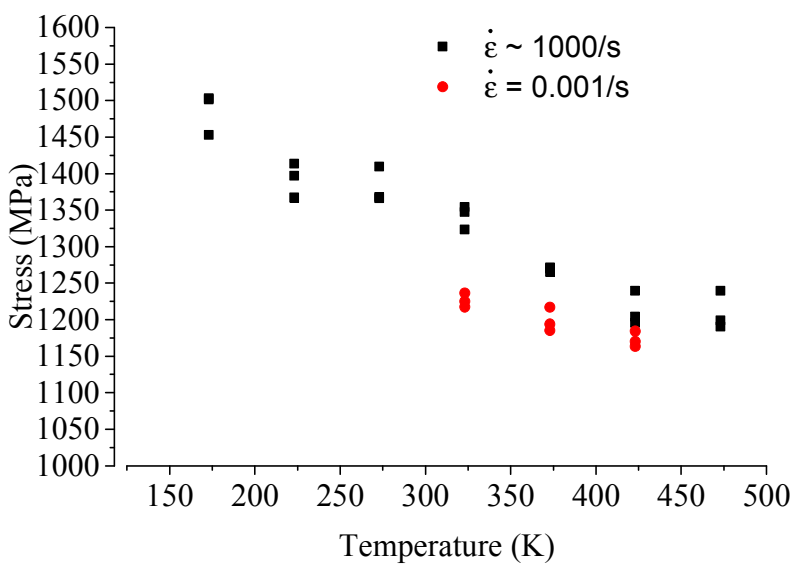

Fig. 4. Stress vs. Temperature (high strength steel).

The Johnson-Cook model is a phenomenological model describing material behaviour through the Eq. (1) [1].

$$
\sigma\left(\varepsilon_{p}, \dot{\varepsilon}_{p}, T\right)=\left(A+B \varepsilon^{n}\right)\left[1+C \log \left(\frac{\dot{\varepsilon}}{\dot{\varepsilon}_{0}}\right)\right]\left(1-T^{* m}\right)
$$

where $A, B, C, n$ and $m$ are the model parameters and $T^{*}$ is the homologous temperature. The parameters $A, B$ and $n$ are determined by fitting stress vs. plastic strain curve at defined conditions $\dot{\varepsilon}_{0}, T_{0}$. Effects of the strain rate and temperature are taken into account in the second and 
third terms, by taking stress value at a fixed level of plastic strain. Therefore, the parameters are easy to identify although this model presents some limits. Firstly, as strain rate and temperature effects are separated, no coupling effect can be taken into account. Moreover, no modification of the hardening as well as no temperature below the reference temperature (in this work, room temperature) can be represented by this model.

\subsection{Rusinek-Klepaczko constitutive model}

This semi-physical model [3] is based on the theory of dislocations and decomposes the stress in an internal stress and an effective stress, Eq. (2).

$$
\sigma\left(\varepsilon_{p}, \dot{\varepsilon}_{p}, T\right)=\frac{E(T)}{E_{0}}\left[\sigma_{\mu}\left(\varepsilon_{p}, \dot{\varepsilon}_{p}, T\right)+\sigma^{*}\left(\dot{\varepsilon}_{p}, T\right)\right]
$$

Where $E(T)$ and $E_{0}$ represent the Young Modulus as function of the temperature and its value at $0 \mathrm{~K}$. Although this value can vary, the evolution of Young modulus of steels [4] is assumed to be low enough (between 173 and $473 \mathrm{~K}$ ) to be neglected. The internal stress is expressed by the Eq. (3):

$$
\sigma_{\mu}\left(\varepsilon_{p}, \dot{\varepsilon}_{p}, T\right)=B\left(\dot{\varepsilon}_{p}, T\right)\left(\varepsilon+\varepsilon_{0}\right)^{n\left(\dot{\varepsilon}_{p}, T\right)}
$$

Where $B$ and $n$ are strain rate and temperature hardening coefficients. $\varepsilon_{0}$ is the strain level defining the yield stress. Eq. (4) and (5) show how are defined $B$ and $n$ :

$$
\begin{aligned}
& B\left(\dot{\varepsilon}_{p}, T\right)=B_{0}\left[\left(\frac{T}{T_{m}}\right) \log \left(\frac{\dot{\varepsilon}_{\text {max }}}{\dot{\varepsilon}}\right)\right]^{-v} \\
& n\left(\dot{\varepsilon}_{p}, T\right)=n_{0}\left\langle 1-D_{2}\left(\frac{T}{T_{m}}\right) \log \left(\frac{\dot{\varepsilon}}{\dot{\varepsilon}_{\min }}\right)\right\rangle
\end{aligned}
$$

Finally, the effective stress is given by the Eq. (6).

$$
\sigma^{*}\left(\dot{\varepsilon}_{p}, T\right)=\sigma_{0}^{*}\left\langle 1-D_{1}\left(\frac{T}{T_{m}}\right) \log \left(\frac{\dot{\varepsilon}_{\max }}{\dot{\varepsilon}}\right)\right\rangle^{m^{*}}
$$

Where $B_{0}, v, n_{0}, D_{2}, \sigma_{0}{ }^{*}, D_{1}$ and $m^{*}$ are the model parameters, $\dot{\varepsilon}_{\text {max }}$ and $\dot{\varepsilon}_{\text {min }}$ are its limits of validity. The operator $\langle x\rangle=x$ if $x>0$ and $\langle x\rangle=0$ otherwise. The internal stress represents the evolution of the dislocation density (creation and annihilation) which is affected by strain (hardening) but also by strain rate and temperature [5]. At the opposite, the effective stress describes an instantaneous effect. From a physical point of view, it represents the difficulty of a dislocation to overcome an obstacle. This additional stress tends to vanish as the temperature increases and increases when the strain rate increases [4]. Therefore, the model neglects this stress at low strain rates for room and higher temperatures.

The first step of the identification of the parameters allows defining $D_{l}$, such as the effective stress is equal to zero at low strain rate $\left(10^{-3} \mathrm{~s}^{-1}\right.$ in the present case $)$ and $293 \mathrm{~K}$ as suggested by the Eq. (7).

$$
D_{1}=\left[\left(\frac{293}{T_{m}}\right) \log \left(\frac{\dot{\varepsilon}_{\max }}{0.001}\right)\right]^{-1}
$$

Assuming that the effective stress is equal to zero at low strain rate, it allows to determine a first approximation of $B(0.001,293)$ and $n(0.001,293)$. As the effective stress corresponds to an instantaneous effect and the internal stress to a history effect, the next step of the identification assumes that at a given strain level (below 0.1 to assume isothermal conditions), the evolution of stress is mainly due to the thermal activation process. Then the Eq. (8) allows to determine the values of $\sigma_{0}{ }^{*}$ and $m$.

$$
\sigma_{0}^{*}\left(\dot{\varepsilon}_{p}, T\right)=\sigma\left(\varepsilon_{p}, \dot{\varepsilon}_{p}, T\right)-\sigma\left(\varepsilon_{p}, 0.001,293\right)
$$

Once the effective stress is defined, values of $B\left(\dot{\varepsilon}_{p}, T\right)$ and $n\left(\dot{\varepsilon}_{p}, T\right)$ for different sets of conditions can be identified. Finally, Eq. (4) and (5) are used to determine $B_{0}, v, n_{0}$ and $D_{2}$.

\section{Evaluation and comparison of the constitutive models}

\subsection{Johnson-Cook model}

A Comparison between experiments and the JohnsonCook model is shown in Fig. 5 and Fig. 6, for both steels. As the strain rate varies during dynamic experiments, an average value is used.

Hardening is well represented by the model in quasistatic conditions, meaning that the first terms of Eq (1) provides a good description of the materials behaviour in these conditions. Nevertheless, in the dynamic regime, one can observe that the model does not fit experimental data and has a trend to deviate for the highest strain rate. The Johnson-Cook model assumes a linear sensitivity of the stress as a function of the logarithm of the strain rates. As shown in Fig. 1 and Fig. 2, strain rate sensitivity is not linear for these steels, but increase for dynamic cases. This explains why this model does not give a good description of materials behaviour for dynamic cases. This problem is especially relevant for the structural steel, as the strain sensitivity is higher than for the high strength steel.

Even if experiments at various temperatures were performed at two distinct strain rates, the identification of the third term of Eq (1) has been achieved using high strain rate condition as more data were available in this regime. The comparison between the model and experiments is shown on the Fig. 7 and Fig. 8. Experiments at various temperatures were performed at an average strain rate of about $1700 / \mathrm{s}$. Regarding the structural steel, as shown previously, the model cannot describe the material behavior at this strain rate. In order to determine if the temperature sensitivity can be represented by this model, the second term of Eq (1) (strain rate sensitivity) has been replaced by a unique coefficient in the cases presented in Fig. 7. As shown in this figure, this model follows quite well the temperature sensitivity for dynamic cases. Nevertheless, one can observe on the Fig. 3 and Fig. 4 that these steels do not exhibit the same temperature sensitivity in quasi-static 
conditions and in dynamic conditions. Therefore, this model does not permit to describe temperature dependency of the mechanical behaviour of the structural steel over the whole range of the studied strain rates.

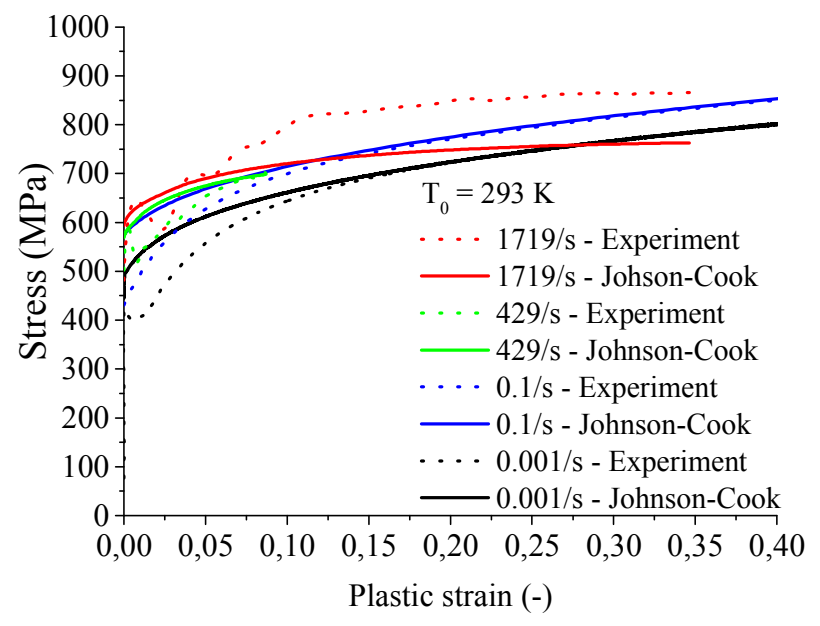

Fig. 5. Stress vs. Plastic strain for different strain rate (structural steel).

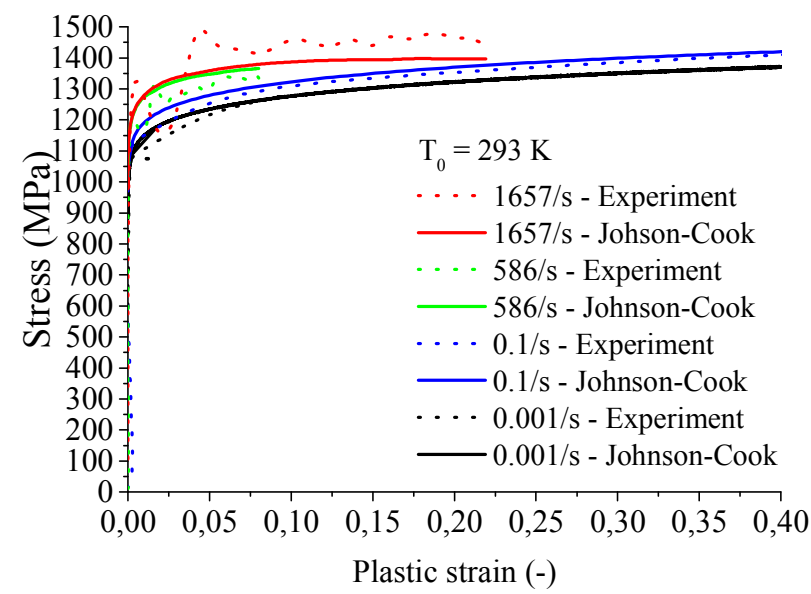

Fig. 6. Stress vs. Plastic strain for different strain rate (high strength steel).

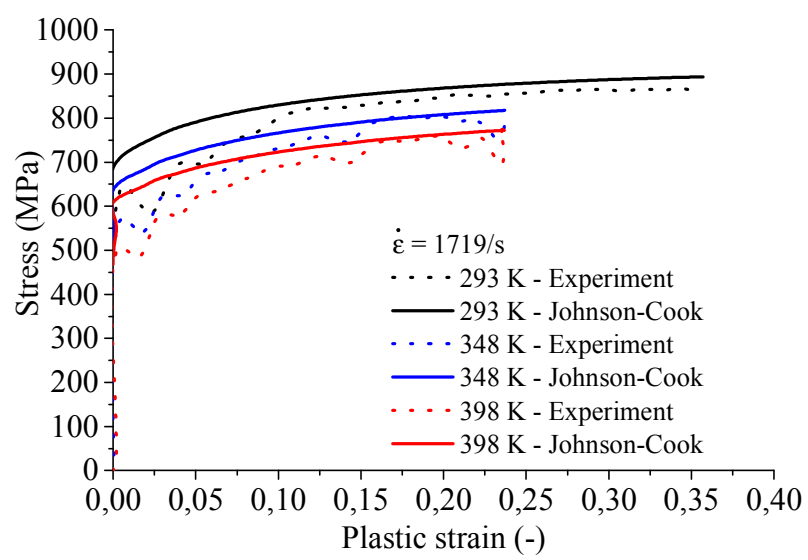

Fig. 7. Stress vs. plastic strain for different temperature (structural steel).

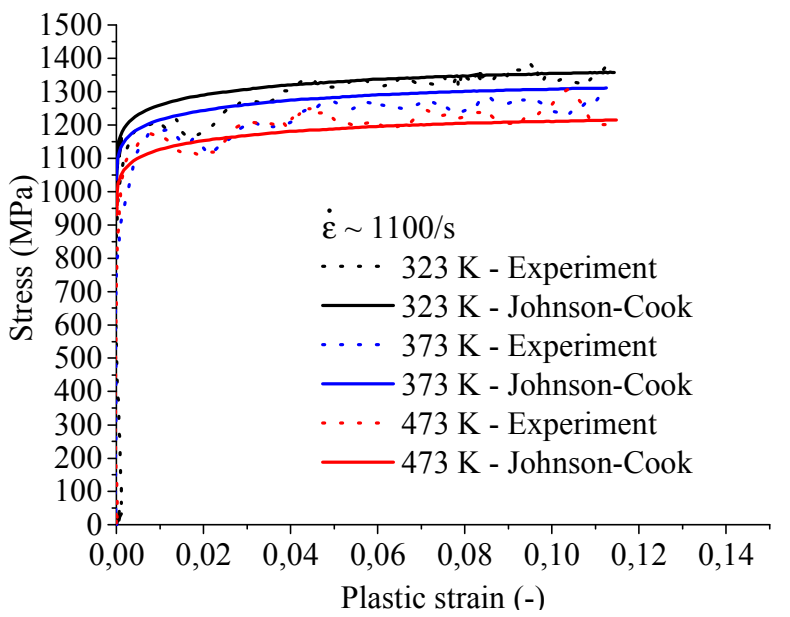

Fig. 8. Stress vs. plastic strain for different temperature (high strength steel).

\subsection{Rusinek-Klepaczko model}

A comparison between experiments at different strain rates and the model is shown on the Fig. 9 and Fig. 10. Regarding the structural steel, this model allows to represent non-linear sensitivity, with a lower sensitivity in quasi-static than in dynamic. Although, the sensitivity in quasi-static is too low to represent experiment performed at $0.1 / \mathrm{s}$, this model provides good representation of the experiments in most of the case studied. Regarding the high strength steel, this model provides a better representation than the Johnson-Cook model.

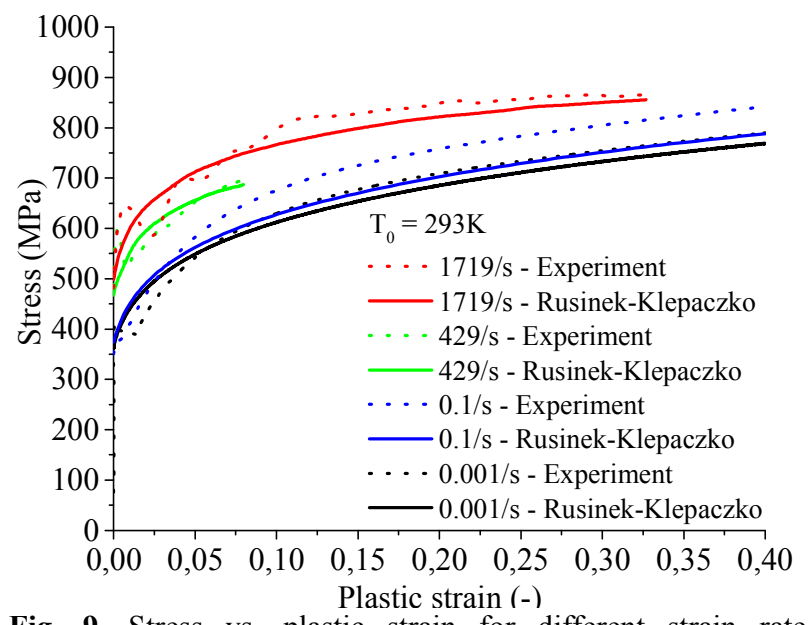

Fig. 9. Stress vs. plastic strain for different strain rate (structural steel). 


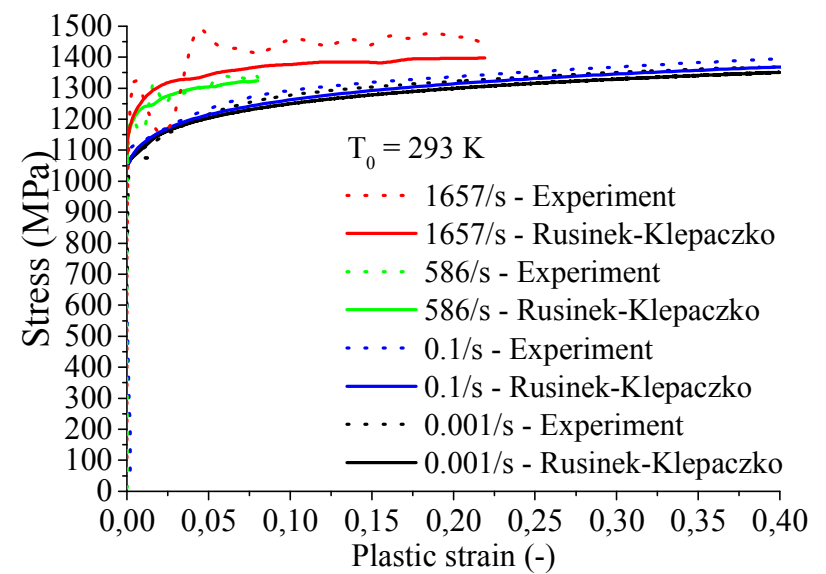

Fig. 10. Stress vs. plastic strain for different strain rate (high strength steel).

Regarding experiment at various temperatures, this model provides, for both steels, a good description. The stress is slightly over-estimated for the structural steel at $173 \mathrm{~K}$, but is well represented at other temperatures. Regarding the high strength steel, all temperatures of the range studied, including the case at $173 \mathrm{~K}$ are well represented by this model.

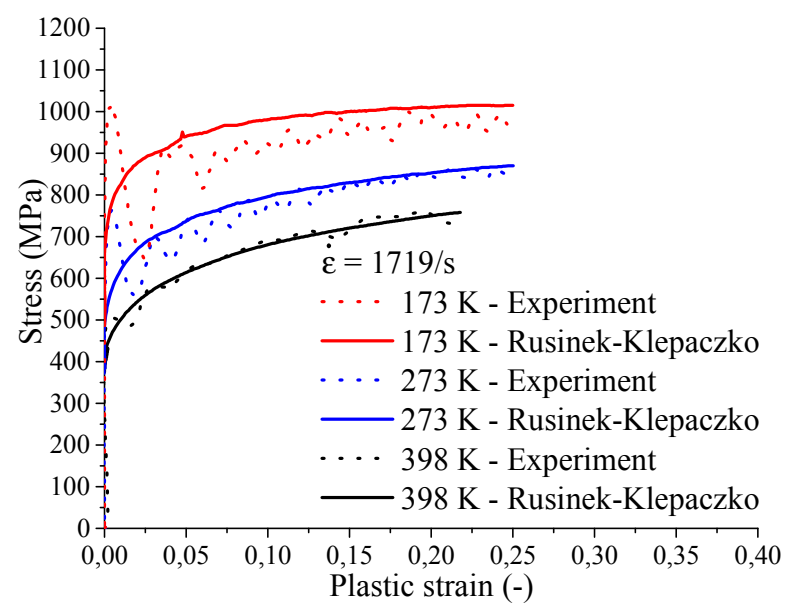

Fig. 11. Stress vs. plastic strain for different temperature (structural steel).

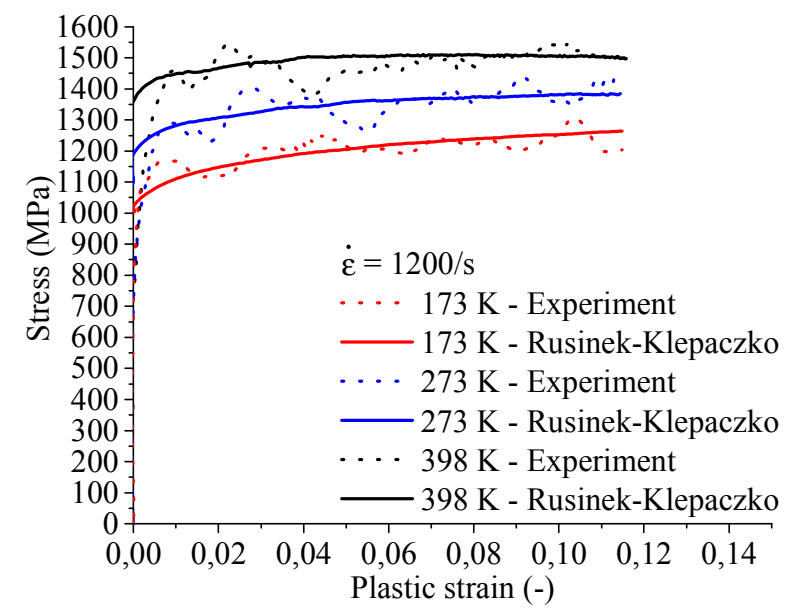

Fig. 12. Stress vs. plastic strain for different temperature (high strength steel).

\section{Conclusion}

The mechanical behaviours of a structural steel and a high strength steel has been studied, with experiments performed at various strain rates varying from $10^{-3} \mathrm{~s}^{-1}$ to $10^{3} \mathrm{~s}^{-1}$ and various temperature going from $173 \mathrm{~K}$ to 473 $\mathrm{K}$. The parameters of the constitutive relations were identified and the results were compared to experiments. The first one is the Johnson-Cook constitutive model. This model allows a good description of temperature sensitivity for both steel, but only for temperature above its reference temperature (293 $\mathrm{K}$ in this work). Furthermore, it assumes a linear strain rate sensitivity, which is not the case of the materials studied. Therefore, a good representation of the dynamic and quasi-static cases cannot be obtained with this model. The second model studied is the Rusinek-Klepaczko constitutive model. At the opposite of the previous model, it is a semi-physical model and allows a better representation of strain rate sensitivity for both materials. Moreover, temperature sensitivity is also well represented, including for experiments performed at temperatures below $293 \mathrm{~K}$.

\section{References}

1. G.R. Johnson, W.H. Cook, (1983)

2. A. Rusinek, R. Bernier, R. Matadi Boubimba, M. Klosak, T. Jankowiak, G. Voyiadjis, Polymer Testing, 65, 1-9, (2018)

4. A. Rusinek, J.R. Klepaczko, Int. J. of Plasticity, 17, 87-115, (2000)

4. J. Klepaczko, A. Rusinek, J.A. Rodriguez-Martinez, Pecherski. R.B., A. Arias, Mech. of Mat., 41, 599621 (2009)

5. J. Klepaczko, Mat. Science and Eng., 18, 121-135, (1975) 
